\title{
Ramos de Inervación y Distribución del Nervio Femoral en el Músculo Cuádriceps Femoral de Individuos Brasileños
}

\author{
Innervation Branches and Distribution of the Femoral Nerve \\ in the Quadriceps Femoral Muscle in Brazilian Individuals
}

Alveal-Mellado, D. ${ }^{1}$; Sousa-Rodrigues, C. F. ${ }^{2}$ \& Olave, E. ${ }^{3}$

ALVEAL-MELLADO, D.; SOUSA-RODRIGUES, C. F. \& OLAVE, E. Ramos de inervación y distribución del nervio femoral en el músculo cuádriceps femoral de individuos brasileños. Int. J. Morphol., 37(2):712-718, 2019.

RESUMEN: El nervio femoral (NF) se describe originándose desde el plexo lumbar (L2, L3 y L4) y en su recorrido emite ramos destinados a cada una de las porciones del músculo cuádriceps femoral (mCF), los cuales nacen de forma aislada o bien, a partir de troncos comunes. El detalle de la distribución del NF en el mCF, permite disminuir riesgos asociados a diferentes intervenciones quirúrgicas llevadas a cabo en la zona anterior del muslo. Con el propósito describir la distribución del NF en los componentes del mCf. Se utilizaron 15 miembros inferiores formolizados, 10 del lado izquierdo y 5 del lado derecho, de individuos adultos, Brasileños, localizados en los Laboratorios de Anatomía de la Universidade Estadual de Ciências da Saúde de Alagoas (UNCISAL), Maceió, Brasil. El NF se clasificó en cuatro tipos de acuerdo a su ramificación y distribución. El Tipo II se subdividió en 3 subtipos y se presentó en $60 \%$ de las muestras y el tipo III en $20 \%$. El NF se dividió de medial a lateral hasta en 5 ramos (R1,R2,R3,R4,R5), donde el R1 fue el más medial. El R1 dio origen en promedio a 2,47 ramos secundarios (Rs) y a 2,58 ramos terciarios (Rt), en 13,3\% el R1 no emitió Rs. En 73,3\% inervó a sólo a un componente del mCF; el R2 dio origen en promedio a 3,93 Rs y a 3,58 Rt. En 26,7 \% inervó a sólo a un componente del mCF; el R3 dio origen en promedio a 3,33 Rs y a 2,0 Rt. En 80 \% inervó a sólo a un componente del mCF. La distribución de R4 y R5 se muestran en el texto. Resultados biométricos de origen, diámetro y longitud de los ramos mencionados son mostrados en tablas. Los datos obtenidos en esta investigación complementan el conocimiento de la anatomía regional, pudiendo ser utilizados por la clínica quirúrgica y para efectuar tratamientos que mejoren trastornos neurológicos que afectan a la región.

PALABRAS CLAVE: Anatomía, Nervio femoral, biometría, músculo cuádriceps femoral.

\section{INTRODUCCIÓN}

Clásicamente el nervio femoral (NF) se describe originándose desde el plexo lumbar (L2, L3 y L4). En su recorrido, distal al ligamento inguinal, emite 4 ramos destinados a cada una de las porciones del músculo cuádriceps femoral (mCF), los cuales nacen de forma aislada o bien, a partir de troncos comunes (Testut \& Latarjet, 1972; Rouvière \& Delmas, 2005).

La comprensión detallada de la distribución del NF en el mCF, permite al cirujano disminuir los riesgos, asociados a diferentes intervenciones quirúrgicas llevadas a cabo en la zona anterior del muslo (Grob et al., 2015a; Ghijselings et al., 2017), así como también mejorar la eficacia de las inyecciones destinadas a disminuir la espasticidad en diferentes trastornos neurológicos que afecten la zona (Sung et $a l ., 2003)$. Además es relevante a la hora de realizar inter- venciones que utilizan injertos nerviosos, para recuperar la función en pacientes con daño neurológico (Barbe et al., 2011; Goubier et al., 2012; Tung et al., 2012; Brown et al., 2013).

El propósito del presente estudio, que describir la forma en que se distribuyen los ramos del NF en los componentes del mCf. Además, determinar las distancias, en relación a puntos anatómicos relevantes, en que se subdividen, ramifican e interactúan con la musculatura.

\section{MATERIAL Y MÉTODO}

Se utilizaron 15 miembros inferiores formolizados, 10 del lado izquierdo y 5 del lado derecho, de individuos

\footnotetext{
${ }^{1}$ Programa de Magister en Ciencias, mención Morfología, Universidad de La Frontera, Temuco, Chile.

${ }^{2}$ Universidade de Ciências da Saude de Alagoas, Maceió, Brasil

${ }^{3}$ Facultad de Medicina, Universidad de La Frontera, Temuco, Chile.
} 
Brasileños adultos, 14 de ellos de sexo masculino. El estudio se llevó a cabo en los laboratorios de Anatomía de la Universidade Estadual de Ciências da Saúde de Alagoas (UNCISAL), en la ciudad de Maceió, Brasil.

Se realizó una disección por planos en la zona medial, anterior y lateral del muslo. Comenzando con una incisión vertical, ligeramente medial a la espina ilíaca anterosuperior (EIAS), se removió la inserción del ligamento inguinal, el cual fue reflejado hacia medial. Se identificó al nervio femoral (NF) en su recorrido profundo a este ligamento, a nivel de la base del triángulo femoral y lateral a los vasos femorales.

Se desinsertó distalmente el músculo sartorio, removiendo el ramo correspondiente del NF. Además se removió la inserción distal del músculo recto femoral y se reflejó hacia proximal y lateral, preservando sus ramos nerviosos.

Tras separar las estructuras adiposas y fasciales se prescindió de los elementos vasculares y se realizó el seguimiento de los ramos que emite el NF para los músculos: recto femoral (mRf), vasto medial $(\mathrm{mVm})$, vasto intermedio (mVint) y vasto lateral (mVlat), hasta su ingreso al plano muscular (punto motor).

Completada la disección se realizó la esquematización de la muestra, considerando los siguientes criterios:

a) El ramo del NF ubicado más hacia proximal y dirigido hacia el mCF se consideró como el primario (R1).

b) De existir una bifurcación o trifurcación, se consideró como R1 el ubicado más próximo hacia la línea mediana del cuerpo, distinguiendo los siguientes como R2, $\mathrm{R} 3, \mathrm{R} 4$, etc.

c) Se identificó el ramo primario hasta su penetración en el vientre muscular, diferenciándolo de ramos secundarios (Rs), terciarios (Rt) y cuaternarios (Rc) según:

a. Diámetro .

b. Número de divisiones .

c. Longitud.

Posteriormente se analizó la distribución y el origen de los ramos para los diferentes componentes del músculo cuádriceps (mRf; $\mathrm{mVm}$; mVint y mVlat). Luego se procedió a realizar las siguientes mediciones utilizando un cáliper marca Mitutoyo de 0,05 mm de precisión:

a) Longitud del muslo: Distancia desde una línea horizontal, que pasa por la EIAS, hasta una línea horizontal que pasa entre los puntos más prominentes de los cóndilos femorales. b) Diámetro de los ramos primarios: Medido a partir de la ramificación del NF en su recorrido hacia el mCF.

c) Punto de origen de los ramos primarios: Distancia vertical desde la horizontal que pasa por las EIAS, hasta una línea horizontal que pasa por el origen del ramo primario.

\section{RESULTADOS}

Distal al ligamento inguinal, el NF emitió ramos para cada uno de los cuatro músculos que componen el mCF. En base a la forma que adoptó el nervio al ramificarse y la porción muscular inervada, se establecieron cuatro tipos de divisiones (Figs. 1 y 2):

Tipo I: Un ramo medial del NF inervó exclusivamente al $\mathrm{mVm}$ y la inervación de los músculos mVint, mVlat y mRf provino de ramos originados a partir de un tronco común lateral (Figs. 1A y 2). Se presentó en 2 casos (13,33 \%).

Tipo IIa: La inervación de los músculos mVm y mVint provino de ramos originados a partir un tronco común medial del NF. Un ramo intermedio del NF inervó al mVlat y un ramo lateral inervó al mRf (Fig. 1B). Se Identificaron 2 ca$\operatorname{sos}(13,33 \%)$.

Tipo IIb: Un ramo medial del NF inervó al $\mathrm{mVm}$. La inervación de los músculos mVint y mVlat provino de ramos originados a partir de un tronco común intermedio. Finalmente un ramo lateral inervó al mRf (Figs. 1C y 3). Estuvo presente en 6 casos (40\%).

Tipo IIc: Un ramo medial del NF inervó al $\mathrm{mVm}$. Un ramo intermedio inervó tanto al $\mathrm{mVm}$ como al mVint. Y por último, un tronco común lateral originó ramos para la inervación del mVint, mVlat y mRf (Fig. 1D). Estuvo presente en un caso $(6,66 \%)$.

Tipo III: El NF se dividió a un mismo nivel en cuatro ramos, de medial a lateral, para el $\mathrm{mVm}$, mVint, mVlat y mRf (Fig. 1E). Este tipo se presentó en 3 casos (20\%).

Tipo IV: El NF dio origen a cinco ramos, con dos ramos dirigidos hacia el mRf y los tres restantes a cada una de las otras cabezas del mCf (Fig. 1F). Estuvo presente en un caso $(6,66 \%)$.

Según los criterios de clasificación y la nomenclatura utilizada en el presente artículo, el NF presentó hasta 5 ramos, siendo éstos denominados como R1, R2, R3, R4 y $\mathrm{R} 5$. El punto de origen, diámetro y longitud de cada ramo se muestra en la Tabla I. 

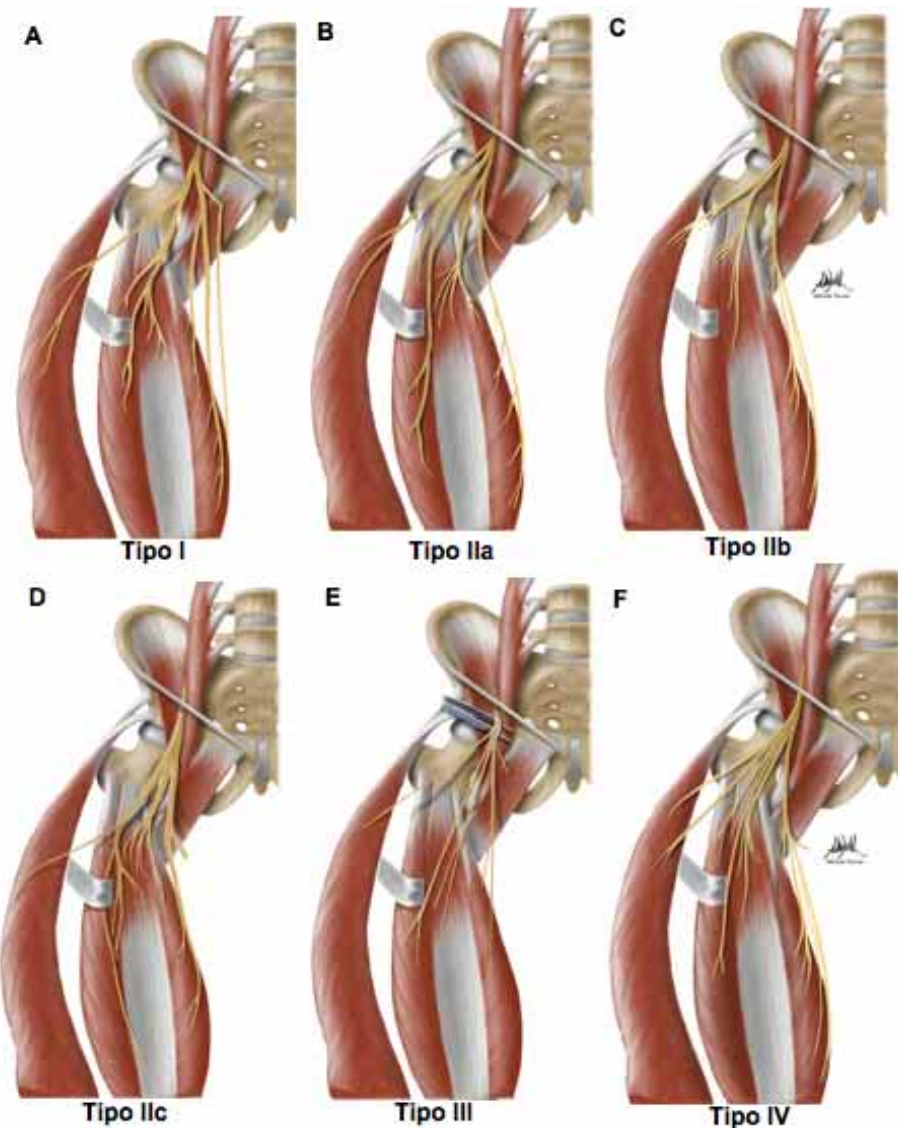

Fig. 1. Patrones de división del nervio femoral (NF) en el músculo cuádriceps femoral (mCf).

La longitud promedio del muslo fue de $461,47 \pm$ $28,72 \mathrm{~mm}$.

El ramo R1 se originó a 70,51 $\pm 12,95 \mathrm{~mm}$ de la EIAS, su diámetro fue de $2,14 \pm 1,08 \mathrm{~mm}$ y tuvo una longitud promedio de 175,24 $\pm 88,32 \mathrm{~mm}$ (Tabla I). En su recorrido hacia el vientre muscular originó en promedio $2,47 \pm 1,64$ ramos secundarios (Rs) (Tabla II). En dos casos $(13,33 \%)$ no emitió Rs. En otro caso $(6,67 \%)$ emitió 6 de ellos. La emisión de ramos terciarios (Rt) estuvo presente en 12 muestras (80\%), promediando 2,58 $\pm 1,38$ de éstos ramos. En una muestra $(6,67$ $\%)$ estuvo presente la emisión de un ramo cuaternario (Rc) .

Este R1 entregó inervación exclusiva a una porción del mCf en 11 casos $(73,33 \%)$, seis de ellos correspondieron al $\mathrm{mVm}$ y cinco al mRf. De manera simultánea inervó 2 músculos en cuatro casos $(26,67 \%)$, en tres ocasiones al $\mathrm{mVm}$ y mVint, y en una ocasión al mVm y mRf (Tabla III).

El ramo R2 se originó a 75,70 \pm 14,99 mm de la EIAS, presentó un diámetro de $2,48 \pm 0,88 \mathrm{~mm}$ y una longitud pro-

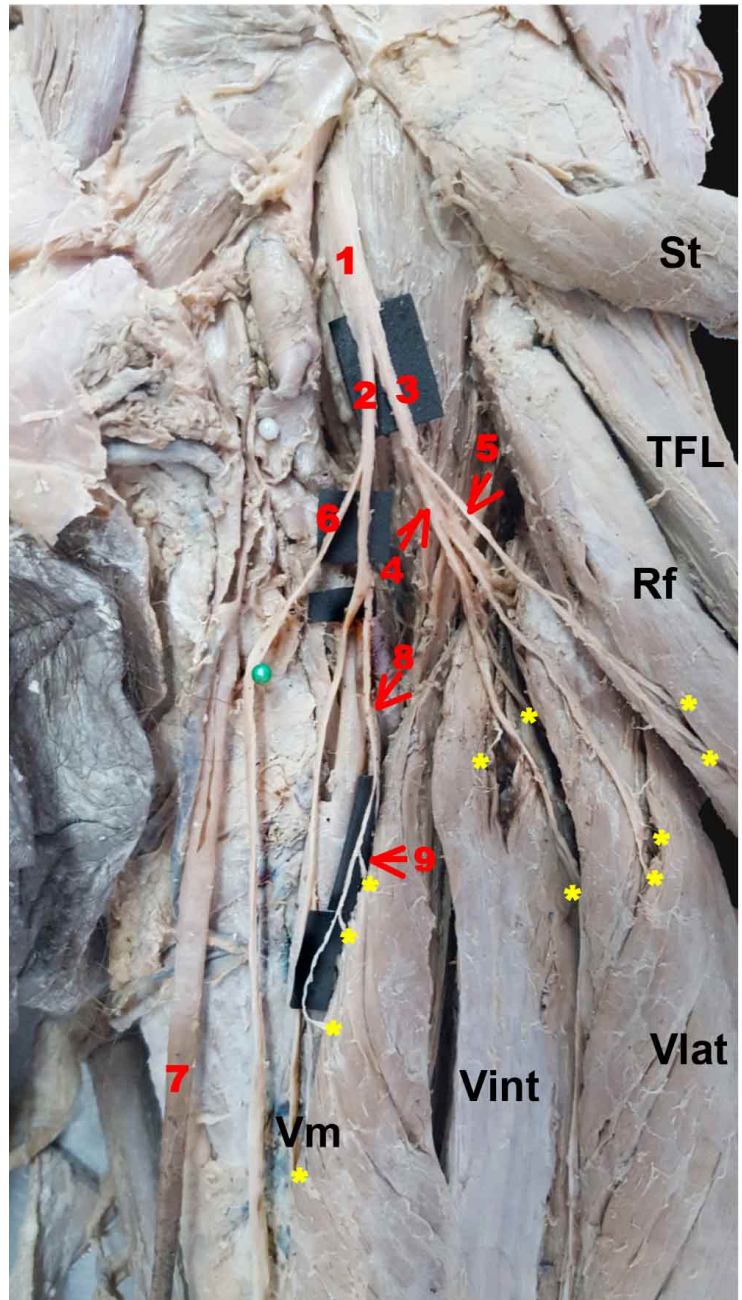

Fig. 2. División tipo I del NF en un muslo izquierdo. 1: Nervio Femoral, 2: Ramo dirigido al mVm (R1). 3: Tronco común lateral, 4: Ramo dirigido a mVint y mVlat (R2), 5: Ramo para el mRf (R3), 6: Nervio Safeno, 7: Arteria femoral profunda, 8: Ramo secundario de R1 (R1S1), 9: Ramo terciario de R1 (R1S1T1), St: Músculo sartorio, TFL: Músculo tensor fascia Lata, Rf: Músculo recto femoral, Vlat: Músculo vasto lateral, Vint: Músculo vasto intermedio, Vm: Músculo vasto medial. Los puntos motores aparecen destacados en asteriscos amarillos.

medio de 152,66 $\pm 58,80 \mathrm{~mm}$ (Tabla I). Emitió en su recorrido 3,93 $\pm 2,43 \mathrm{Rs}$, con un mínimo de un Rs en dos casos $(13,33 \%)$ y un máximo de 10 Rs en otro $(6,67 \%)$. Este ramo emitió al menos un Rt en 12 muestras $(80 \%$ ), promediando 3,58 $\pm 3,28$ Rt. Por último, 4 muestras $(26,67 \%)$ emitieron 1,00 $\pm 0,82$ Rc en promedio (Tabla II).

El destino de $\mathrm{R} 2$ fue muy variable en el $\mathrm{mCf}$, inervando exclusivamente un músculo en 4 casos 


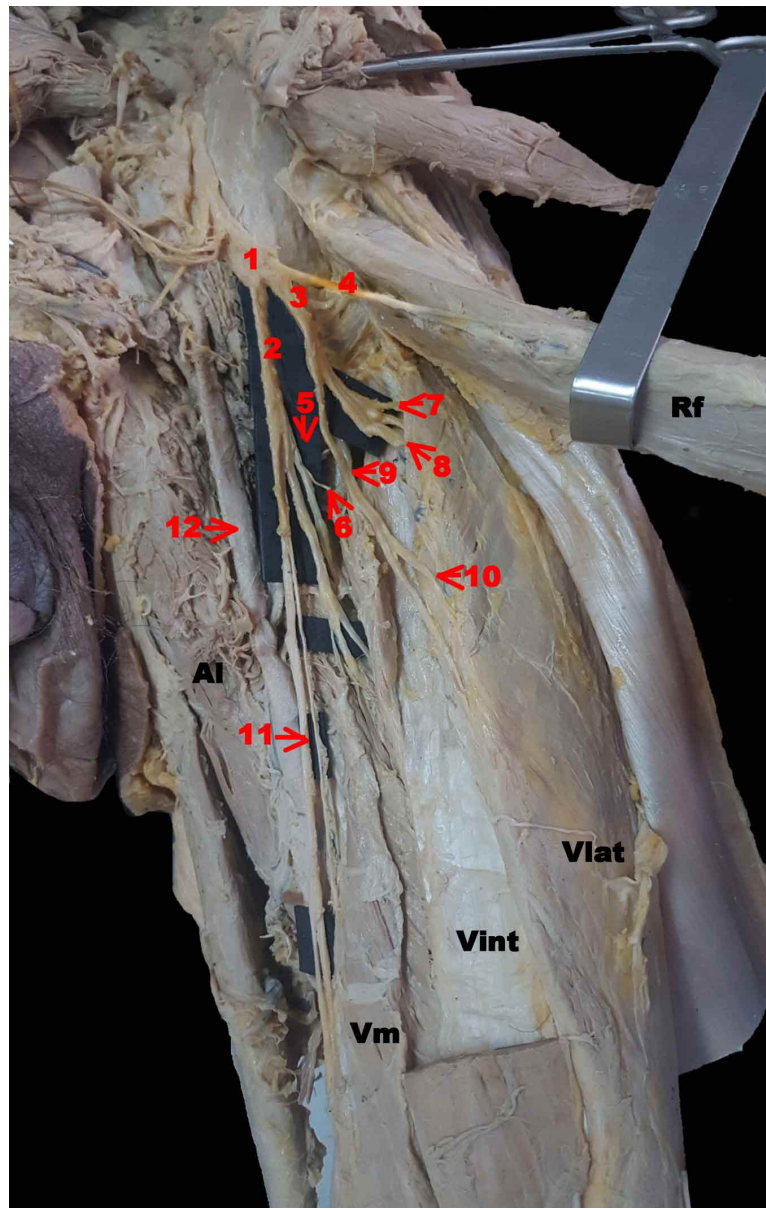

Fig. 3. Distribución tipo Ilb del NF en un muslo izquierdo.1: Nervio femoral, 2: Ramo dirigido al $\mathrm{mVm}$ (R1), 3: Ramo dirigido al mVint y mVlat (R2), 4: Ramo dirigido al mRf (R3), 5: Ramo secundario de R1 (R1S1) dirigido al $\mathrm{mVm}, 6$ : Ramo terciario de R1 (R1S1T1) dirigido al mVm, 7: Ramo secundario de R2 (R2S1) dirigido al mVlat, 8: Ramo secundario de R2 (R2S2) dirigido al mVlat, 9: Ramo secundario de R2 (R2S3) dirigido al mVint. 10: Ramo terciario de R2 (R2S3T1) dirigido al mVint, 11: Nervio safeno, 12: Arteria femoral profunda, Al: Músculo aductor largo, Vm: Músculo vasto medial, Vint: Músculo vasto intermedio, Vlat: Músculo vasto lateral, Rf: Músculo recto femoral.
(26,67\%), dos músculos en 9 casos $(60 \%)$ y tres músculos en 2 casos $(13,33)$. Los músculos inervados se detallan en la Tabla III.

El tercero de los ramos (R3) se originó a 81, 95 \pm 17,58 mm de la EIAS, presentó un diámetro de 2,06 $\pm 1,19 \mathrm{~mm}$ y tuvo una longitud de 159,80 $\pm 85,41 \mathrm{~mm}$ (Tabla I). Dio origen a 3,33 $\pm 1,95 \mathrm{Rs}$ en promedio, variando desde un Rs en dos casos $(13,33 \%)$ hasta 8 Rs en otro caso $(6,67 \%)$. Este $\mathrm{R} 3$, en 10 muestras $(66,67 \%)$ se ramificó hasta emitir 2,00 \pm 1,15 Rt y en 3 muestras $(20 \%)$ emitió 2,00 $\pm 1,73$ Rc (Tabla II).

En 12 muestras (80 \%) R3 tuvo como destino la inervación de un solo músculo y en 3 ocasiones (20\%) se dirigió a dos músculos de forma simultánea. Estos músculos se detallan en la Tabla III.

En las 8 muestras en que estuvo presente, R4 se originó a 89, $37 \pm 13,41 \mathrm{~mm}$ de la EIAS, presentó un diámetro de $2,23 \pm 0,57 \mathrm{~mm}$ y tuvo una longitud promedio de 152,43 \pm $76,97 \mathrm{~mm}$ (Tabla I). A partir de un ramo primario se originaron 3,00 $\pm 1,95 \mathrm{Rs}$, penetrando directamente en el músculo en una ocasión $(12,5 \%)$ y observándose la emisión de hasta siete Rs en un caso (12,5\%). Así mismo, emitió 3,50 \pm 1,64 Rt, sin emisiones de Rc (Tabla II).

La inervación que entregó R4 correspondió a un músculo de forma exclusiva en 5 ocasiones $(62,5 \%)$ y a dos músculos de forma simultánea en 3 ocasiones (37,5\%), los cuales se muestran detallados en la Tabla III.

El ramo R5 estuvo presente en un caso. Se originó a 101,93 mm de la EIAS, presentó un diámetro de 1,65 mm y una longitud de 94,22 mm (Tabla I). Emitió 3 Rs y 4 Rc (Tabla II). Inervó simultáneamente al mVint y al mVm (Tabla III).

Tabla I. Promedio de los puntos de origen, diámetro y longitud de los ramos primarios.

\begin{tabular}{ccccc}
\hline Ramos & Punto de origen $(\mathrm{mm})$ & Diámetro $(\mathrm{mm})$ & Longitud $(\mathrm{mm})$ & $\mathrm{N}^{\circ}$ Casos \\
\hline R1 & $70,51 \pm 12,95$ & $2,14 \pm 1,08$ & $175,24 \pm 88,32$ & 15 \\
R2 & $75,70 \pm 14,99$ & $2,48 \pm 0,88$ & $152,66 \pm 58,80$ & 15 \\
R3 & $81,95 \pm 17,58$ & $2,06 \pm 1,19$ & $159,80 \pm 85,41$ & 15 \\
R4 & $89,37 \pm 13,41$ & $2,23 \pm 0,57$ & $152,43 \pm 76,97$ & 8 \\
R5 & 101,93 & 1,65 & 94,22 & 1 \\
\hline
\end{tabular}

Tabla II. Cantidad promedio de Rs, Rt y Rc originados a partir de un ramo primario.

\begin{tabular}{ccccccc}
\hline Ramo Primario & N Rs (DE) & Obs. & N Rt (DE) & Obs. & N Rc (DE) & Obs. Rc \\
\hline R1 & $2,47(1,64)$ & 15 & $2,58(1,38)$ & 12 & $1,00(--)$ & 1 \\
R2 & $3,93(2,43)$ & 15 & $3,58(3,28)$ & 12 & $1,00(0,82)$ & 4 \\
R3 & $3,33(1,95)$ & 15 & $2,00(1,15)$ & 10 & $2,00(1,73)$ & 3 \\
R4 & $3,00(2,14)$ & 8 & $3,50(1,64)$ & 6 & -- & -- \\
R5 & $3,00(--)$ & 1 & $4(-)$ & 1 & -- & -- \\
\hline
\end{tabular}


Tabla III. Destino muscular de los ramos primarios.

\begin{tabular}{|c|c|c|c|c|c|c|}
\hline \multirow[t]{2}{*}{ Ramo } & \multicolumn{2}{|c|}{ Destino 1 músculo } & \multicolumn{2}{|c|}{ Destino 2 músculos } & \multicolumn{2}{|c|}{ Destino 3 músculos } \\
\hline & Músculos & $\mathrm{N}^{\circ}$ casos & Músculos & $\mathrm{N}^{\circ} \operatorname{casos}$ & Mú sculos & $\mathrm{N}^{\circ}$ casos \\
\hline \multirow{4}{*}{$\mathrm{R} 1$} & $\mathrm{mVm}$ & 6 & mVm-mVint & 3 & -- & -- \\
\hline & $\mathrm{mRf}$ & 5 & $\mathrm{mVm}-\mathrm{mRf}$ & 1 & -- & -- \\
\hline & Total & 11 & Total & 4 & -- & -- \\
\hline & $\mathrm{mVm}$ & 1 & $\mathrm{mVm}$-mVint & 3 & mVm-mVint-mVlat & 2 \\
\hline \multirow{4}{*}{$\mathrm{R} 2$} & mVlat & 2 & mVint-mVlat & 5 & -- & -- \\
\hline & $\mathrm{mRf}$ & 1 & mVlat-mRf & 1 & -- & -- \\
\hline & Total & 4 & Total & 9 & Total & 2 \\
\hline & $\mathrm{mVm}$ & 4 & mVm-mVint & 1 & -- & -- \\
\hline \multirow{4}{*}{ R3 } & mVlat & 1 & mVint-mVlat & 2 & -- & -- \\
\hline & $\mathrm{mRf}$ & 7 & 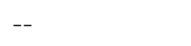 & -- & -- & -- \\
\hline & Total & 12 & Total & 3 & -- & -- \\
\hline & mVint & 3 & $\mathrm{mVm}$-mVint & 1 & -- & -- \\
\hline \multirow[t]{2}{*}{$\mathrm{R} 4$} & $\mathrm{mRf}$ & 2 & mVint-mVlat & 2 & -- & -- \\
\hline & Total & 5 & Total & 3 & -- & -- \\
\hline \multirow{2}{*}{ R5 } & -- & -- & mVint-mVlat & 1 & -- & -- \\
\hline & Total & -- & Total & 1 & -- & -- \\
\hline$\sum \mathrm{R}$ & & 32 & & 22 & -- & -- \\
\hline
\end{tabular}

\section{DISCUSIÓN}

En las muestras analizadas el NF emitió hasta 5 ramos, encontrándose seis tipos distintos de ramificación (Fig. 1). La división en tres ramos, uno medial para el $\mathrm{mVm}$, un tronco común intermedio, del cual emergen ramos para el $\mathrm{mVint}$ y mVlat, y un ramo lateral para el $\mathrm{mRf}$ fue observado con mayor frecuencia (Tipo IIb).

Tal división contrasta con lo presentado por Tung et $a l$, donde el NF se dividió en las ocho muestras analizadas en 3 ramos. La división más medial originó el N. Safeno, la intermedia originó el ramo para el $\mathrm{mVm}$ y mVint a través de un tronco común y la más lateral originó los ramos para el mVlat y mRf, también a través de un tronco común.

El origen común de los ramos hacia el $\mathrm{mVm}$ y mVint (tipo IIa) fue observado un 13,33\% en el presente estudio. Y el origen común para el mVlat y mRf (Tipo IIc) un 6,66 \%.

Mediciones en relación a los puntos de origen de los ramos motores del NF que inervan el mCf son escasas en la literatura. Sólo Sung et al. mencionaron que el ramo destinado al mRf se originó a $107 \pm 17 \mathrm{~mm}$ de la EIAS, distancia mayor a la encontrada en nuestro estudio para el ramo R3 $(81,95 \pm 17,58)$, que la mayoría de las veces se dirigió a ese músculo.

El diámetro y la longitud de los ramos son relevantes al momento de evaluar la compatibilidad de injertos en la reconstrucción nerviosa. En nuestro estudio, el ramo de mayor diámetro fue $\mathrm{R} 2(2,48 \pm 0,88)$ y el de menor fue R5 $(1,65 \mathrm{~mm})$. La literatura reporta gran variabilidad en este sentido. Goubier et al. informaron un diámetro promedio de la porción motora del NF (que incluye inervación a otros músculos además del mCF) de 3,7 mm. Yang \& Morris (1999) describieron un diámetro de 1,8 \pm 0,40 mm correspondiente al ramo destinado al $\mathrm{mRf}$ y Sung et al. reportaron 2,6 $\pm 2,00 \mathrm{~mm}$ para el mismo ramo.

En cuanto a la longitud de cada ramo, en el presente estudio, el de menor longitud fue R5 (94,22 mm) y el de mayor, R1 (175,24 $\pm 88,32 \mathrm{~mm})$. Tung et al. mostraron en sus resultados longitudes inferiores a las nuestras, siendo el ramo para el $\mathrm{mRf}$ el de menor longitud $(49,4 \mathrm{~mm} \pm 9 \mathrm{~mm})$, seguidos por el ramo destinado al mVint $(67 \pm 23 \mathrm{~mm})$, mVlat $(103 \pm 49 \mathrm{~mm})$ y mVm $(113 \pm 31 \mathrm{~mm})$. Barbe et al. reportaron que el ramo hacia el vasto medial midió $174 \pm 8 \mathrm{~mm}$, similar a los 175,24 $\pm 88,32 \mathrm{~mm}$ de longitud de R1, que en un $40 \%$ de las muestras del presente estudio, se dirigió a dicho músculo.

Grob et al. (2015b) observaron que el mVlat está inervado de dos formas; ramos cortos del NF inervan la porción proximal del músculo, mientras que ramos más largos inervan la porción distal. La longitud promedio de los ramos cortos fue de $36 \mathrm{~mm}$ (19-50 mm) y los ramos largos $113 \mathrm{~mm}$ (78-161 mm). En el estudio actual, en 10 ocasiones $(66,67 \%)$, el ramo R2 inervó al mVlat, con una longitud de $152,66 \pm 58,80 \mathrm{~mm}$. 
Yang \& Morris describieron la inervación del mRf, cuyo ramo alcanzó una longitud promedio de $150 \pm 6 \mathrm{~mm}$. Menor variabilidad comparado a los $159,80 \pm 85,41$ que presentó R3 en nuestro estudio, el cual inervó de manera exclusiva dicho músculo en 7 ocasiones $(46,67 \%)$.

El recorrido de un ramo nervioso finaliza al momento de ingresar en la porción muscular, lugar conocido como punto motor (Olave et al., 2009). Un mismo ramo del NF puede originar puntos motores en más de una porción del mCf. En las muestras analizadas, en ocasiones, los ramos primarios inervaron tanto al mVlat como mVint, en otras, tanto al $\mathrm{mVm}$ como al mVint. Esta inervación compartida se corresponde con lo observado por D'Arpa et al. (2016) y Grob et al. (2015a), quienes describieron la inervación del mVint a partir de la división medial del ramo dirigido al mVlat y también a partir de la división lateral del ramo dirigido hacia el $\mathrm{mVm}$.

El NF de manera similar a los bronquios y bronquiolos en el sistema respiratorio, se divide varias veces antes de penetrar en el músculo. En las muestras analizadas, se encontró hasta un ramo cuaternario ( $\mathrm{Rc}$ ), lo que corresponde a 3 divisiones sucesivas a partir un ramo principal. La evidencia disponible entrega escasa información a este respecto.

Nuestro estudio se centró en el seguimiento del NF hasta la porción muscular, sin considerar el recorrido intramuscular. Orduña Valls et al. (2017) describieron que los ramos del NF dirigidos a los músculos vastos, entregan inervación sensitiva a la porción anterior de la articulación de la rodilla a través de recorridos intramusculares. Hallazgos similares habían sido encontrados por Burckett-St. Laurant et al. (2016), lo que refuerza la idea del complejo entramado vasculo-nervioso que existe al interior de la musculatura anterior del muslo.

Finalmente, nuestra investigación entrega datos biométricos que pueden ser utilizados al momento de realizar intervenciones quirúrgicas en la zona anterior del muslo. Sin embargo, deben considerarse las limitaciones relacionadas con las contracturas pos-mortem que alteran la posición de las articulaciones y por lo mismo, disminuyen el grado de exactitud de las mediciones.

ALVEAL-MELLADO, D.; SOUSA-RODRIGUES, C.F. \& OLAVE, E. Innervation branches and distribution of the femoral nerve in the quadriceps femoral muscle in brazilian individuals. Int. J. Morphol., 37(2):712-718, 2019.

SUMMARY: The femoral nerve (FN) is described as originating from the lumbar plexus (L2, L3 and L4) and in its course it emits branches destined to each one of the quadriceps femoral muscle $(\mathrm{QFm})$, which are originated in an isolated way or, from common trunks. The detail of the distribution of the FN in the QFm, allows to diminish risks associated with different surgical interventions carried out in the anterior thigh area. With the purpose of describing the distribution of FN in the QFm components. Fifteen formalized lower limbs were used, 10 on the left side and 5 on the right side of adult individuals, Brazilians, located in the Anatomy Laboratories of the State University of Ciências da Saúde de Alagoas (UNCISAL), Maceió, Brazil. The FN was classified into four types according to its branch and distribution. Type II was subdivided into 3 subtypes and presented in $60 \%$ of the samples and type III in $20 \%$. The FN was divided from medial to lateral in 5 branches (B1, B2, B3, B4, B5), where B1 was the most medial. The $\mathrm{B} 1$ gave rise to an average of 2.47 secondary branches (sB) and to 2.58 tertiary branches $(\mathrm{tB})$, in $13.3 \%$ the $\mathrm{B} 1$ did not emit sB. In $73.3 \%$, only one component of the QFm was invested; B2 gave rise to an average of $3.93 \mathrm{sB}$ and $3.58 \mathrm{tB}$. In $26.7 \%$, it invested only one component of the QFm; B3 gave rise to an average of $3.33 \mathrm{sB}$ and $2.0 \mathrm{tB}$. In $80 \%$, it invested only one component of the QFm. The distribution of B4 and B5 are shown in the text. Biometric results of origin, diameter and length of the mentioned branches are shown in tables. The data obtained in this research complements the knowledge of the regional anatomy, being able to be used by the surgical clinic and to carry out treatments that improve neurological disorders that affect the region.

KEY WORDS: Anatomy, Femoral nerve, biometry, quadriceps femoral muscle.

\section{REFERENCIAS BIBLIOGRÁFICAS}

Barbe, M. F.; Brown, J. M.; Pontari, M. A.; Dean, G. E.; Braverman, A. S. \& Ruggieri, M. R. Feasibility of a femoral nerve motor branch for transfer to the pudendal nerve for restoring continence: a cadaveric study. J. Neurosurg. Spine, 15(5):526-31, 2011.

Brown, J. M.; Barbe, M. F; Albo, M. E. \& Ruggieri, M. R. Anatomic feasibility of restoring bladder capacity and voiding following proximal spinal cord injury using a femoral branch to bilateral pelvic nerve transfer: A cadaver study. J. Neurosurg. Spine, 18(6):598-605, 2013.

Burckett-St. Laurant, D.; Peng, P.; Girón Arango, L.; Niazi, A. U.; Chan, V. W.; Agur, A. \& Perlas, A. The nerves of the adductor canal and the innervation of the knee: an anatomic study. Reg. Anesth. Pain Med., 41(3):321-7, 2016.

D’Arpa, S.; Toia, F.; Brenner, E.; Melloni, C.; Moschella, F. \& Cordova, A. Variability and reliability of the vastus lateralis muscle anatomy. Acta Chir. Belg., 116(4):203-12, 2016.

Ghijselings, S.; Driesen, R.; Simon, J. P. \& Corten, K. Distal extension of the direct anterior approach to the hip: A cadaveric feasibility study. $J$. Arthroplasty, 32(1):300-3, 2017.

Goubier, J. N.; Teboul, F. \& Yeo, S. Transfer of two motor branches of the anterior obturator nerve to the motor portion of the femoral nerve: an anatomical feasibility study. Microsurgery, 32(6):463-5, 2012.

Grob, K.; Monahan, R.; Gilbey, H.; Ackland, T. \& Kuster, M. S. Limitations of the vastus lateralis muscle as a substitute for lost abductor muscle function: an anatomical study. J. Arthroplasty, 30(12):2338-42, 2015 b.

Grob, K.; Monahan, R.; Gilbey, H.; Yap, F.; Filgueira, L. \& Kuster, M. Distal extension of the direct anterior approach to the hip poses risk to neurovascular structures: an anatomical study. J. Bone Joint Surg. Am., 97(2):126-32, $2015 \mathrm{a}$. 
Olave, E.; Gabrielli, C. \& Braga, M. T. T. Innervation patterns and biometric localization of the motor points of the brachial biceps muscle in man. Int. J. Morphol., 27(2):495-501, 2009.

Orduña Valls, J. M.; Vallejo, R.; López Pais, P.; Soto, E.; Torres Rodríguez, D.; Cedeño, D. L.; Tornero Tornero, C; Quintáns Rodríguez, M.; Baluja González, A. \& Álvarez Escudero, J. Anatomic and ultrasonographic evaluation of the knee sensory innervation: a cadaveric study to determine anatomic targets in the treatment of chronic knee pain. Reg. Anesth. Pain Med., 42(1):90-8, 2017.

Rouvière, H. \& Delmas, A. Anatomía Humana: Descriptiva, Topográfica y Funcional. Vol. 3. 11 a ed. Barcelona, Masson, 2005.

Sung, D. H.; Jung, J. Y.; Kim, H. D.; Ha, B. J. \& Ko, Y. J. Motor branch of the rectus femoris: anatomic location for selective motor branch block in stiff-legged gait. Arch. Phys. Med. Rehabil., 84(7):1028-31, 2003.

Testut, L. \& Latarjet, A. Tratado de Anatomía Humana. Vol. 3. $9^{\mathrm{a}}$ ed. Barcelona, Salvat, 1972.

Tung, T. H.; Chao, A. \& Moore, A. M. Obturator nerve transfer for femoral nerve reconstruction: anatomic study and clinical application. Plast. Reconstr. Surg., 130(5):1066-74, 2012.

Yang, D. \& Morris, S. F. Neurovascular anatomy of the rectus femoris muscle related to functioning muscle transfer. Plast. Reconstr. Surg., 104(1):102-6, 1999.

\author{
Dirección para correspondencia: \\ Dr. Enrique Olave \\ Facultad de Medicina \\ Universidad de La Frontera \\ Av. Francisco Salazar 01145 \\ Temuco \\ CHILE
}

Email: enrique.olave@ufrontera.cl

Recibido : 03-01-2019

Aceptado: 22-02-2019 